
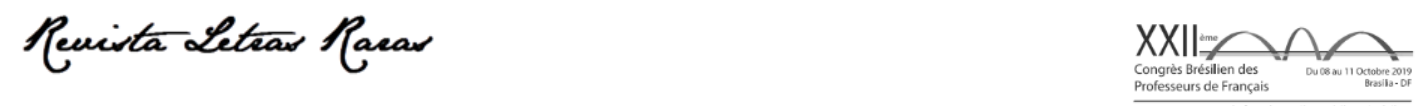

Actes du XXIlème Congrès brésilien des professeurs de français (dans Édition spéciale de la Revue Letras Raras 2020)

ISSN : 2317-2347 - v. 9, Dossier Spécial (2020)

Todo o conteúdo da RLR está licenciado sob Creative Commons Atribuição 4.0 Internacional

\title{
Le calque comme difficulté de traduction dans Véra, récit fantastique de Villiers de l'Isle-Adam
}

\section{Laís Marx Umpierre*}

Licenciée en lettres françaises à l'université fédérale de Rio Grande (FURG), professeur de langue française au « Centro de ensino de línguas estrangeiras » (CELE).

iD https://orcid.org/0000-0003-4687-7601

\section{Luísa Freire ${ }^{* *}$}

Étudiante en lettres françaises et membre du projet de recherche « Literatura Fantástica Francesa e Tradução », mené à l'université fédérale de Rio Grande.

\section{iD https://orcid.org/0000-0002-2415-6320}

\section{Gabriela Jardim da Silva ${ }^{* * *}$}

Titulaire d'un Doctorat en littérature française, enseignante au département de langue et culture françaises de l'université fédérale de Rio Grande (FURG).

iD http://orcid.org/0000-0002-7758-5241

Reçu le13 sep. 2020. Approuvé le 25 oct. 2019.

\section{Comment citer cet article:}

MARX UMPIERRE, Laís ; FREIRE, Luísa. SILVA, Gabriela Jardim. Le calque comme difficulté de traduction dans Véra, récit fantastique de Villiers de L'Isle-Adam. In: CONGRES BRESILIEN DES PROFESSEURS DE FRANÇAIS, 22., 2019, Brasília. Actes du XXIlème Congrès Brésilien des Professeurs de Français. Édition spéciale de la Revue Letras Raras: Campina Grande. EDUFCG. nov. 2020, p. 358-367.

\section{RÉSUMÉ}

Insérée dans le projet de recherche intitulé Literatura fantástica francesa e tradução, mené à l'université fédérale de Rio Grande (FURG) et qui se destine à la promotion auprès du public brésilien de quelques récits de la littérature fantastique française du XIXe siècle, par le moyen de leur analyse et de leur traduction, cette étude a pour but d'exposer le calque comme difficulté de traduction dans Véra (1874), nouvelle d'Auguste Villiers de l'Isle-Adam. Les difficultés de compréhension et/ou de traduction sont considérées comme de véritables obstacles à la transposition de la langue-

$\triangle$ umpierre.lais@gmail.com

**

luisagfreire@gmail.com

gabriela.jardim@furg.br 

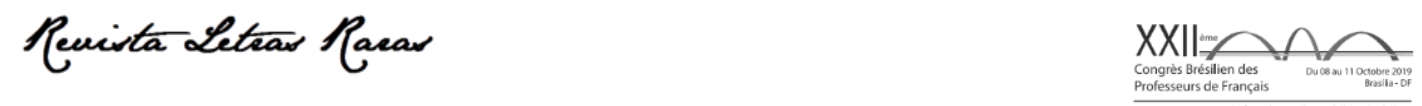

Actes du XXIlème Congrès brésilien des professeurs de français (dans Édition spéciale de la Revue Letras Raras 2020)

ISSN : 2317-2347 - v. 9, Dossier Spécial (2020)

Todo o conteúdo da RLR está licenciado sob Creative Commons Atribuição 4.0 Internacional

source vers la langue-cible. Néanmoins, certains chercheurs se sont penchés sur cette question, à l'instar de Paulo Rónai, dont les écrits ont été étudiés dans le cadre de cette recherche (RÓNAI 1976, 1983a, 1983b) ; la méthodologie sur laquelle nous nous basons est, par conséquent, bibliographique. De nombreux types de difficultés ont été relevés au cours de la traduction de Véra, pourtant, dans ce travail, nous ne nous pencherons que sur le calque. Par l'intermédiaire de l'examen des difficultés concrètes observées, nous envisageons de trouver les solutions les plus adéquates aux occurrences linguistiques pouvant susciter des calques auxquelles nous avons été confrontées. Finalement, après avoir énoncé les spécificités du calque et en avoir fourni des exemples, nous présenterons quelques-unes de nos conclusions, dont l'importance d'unir la théorie à la pratique pour dépasser les problèmes rencontrés au moment de traduire un texte, quel que soit son genre.

MOTS-CLÉS : Traduction (difficultés de); Calque; Littérature française; Villiers de l'Isle-Adam; Véra (1874).

\section{Introduction}

Ce travail a pour dessein d'exposer le calque, procédé linguistique de création d'une construction par emprunt d'une structure à une autre langue, comme difficulté de traduction dans Véra, nouvelle d'Auguste Villiers de I'Isle-Adam, publiée en 1874. Pour ce faire, nous nous appuyons sur une méthodologie bibliographique et nous nous basons sur les études de Paulo Rónai, traducteur et chercheur qui s'est penché sur les difficultés de compréhension et de traduction du français en portugais du Brésil.

À l'occasion de la traduction de Véra, nous avons trouvé de nombreux types de difficultés, cependant, dans cet article, nous ne réfléchissons que sur le calque. Les démarches que nous menons ici visent aux solutions les plus adéquates pour surmonter certaines des barrières auxquelles nous avons été confrontées en ce qui concerne la transposition de la langue-source vers la langue-cible.

Après avoir présenté les spécificités du calque et en avoir fourni deux exemples, l'un dans le domaine de la morphosyntaxe et l'autre dans le domaine de la stylistique, nous exposons quelques-unes de nos conclusions, parmi lesquelles l'importance d'associer la théorie et la pratique pour dépasser les obstacles rencontrés lors d'une traduction.

\section{Le projet de recherche Literatura fantástica francesa e tradução}

Nous avons développé ce travail dans le cadre du projet de recherche intitulé Literatura fantástica francesa e tradução, mené à l'université fédérale de Rio Grande (FURG). Ce projet s'applique à l'examen de quelques nouvelles fantastiques françaises publiées au XIXe siècle.

Nous avons par ailleurs l'intention (a) de promouvoir la diffusion de quelques-unes de ces nouvelles fantastiques au public brésilien par le moyen de leur traduction en portugais et (b) 

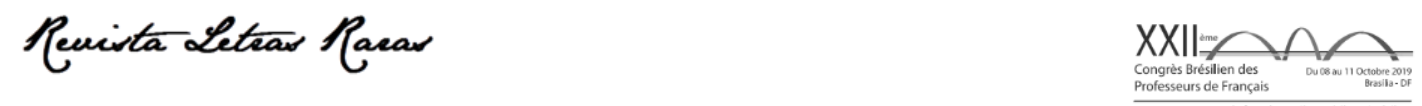

Actes du XXIlème Congrès brésilien des professeurs de français (dans Édition spéciale de la Revue Letras Raras 2020)

ISSN : 2317-2347 - v. 9, Dossier Spécial (2020)

Todo o conteúdo da RLR está licenciado sob Creative Commons Atribuição 4.0 Internacional

d'élaborer des travaux concernant les difficultés de compréhension et/ou de traduction rencontrées lors du processus traductif, dont le calque, sujet de cette étude.

\section{Le calque}

Avant d'en fournir des exemples concrets, nous essayons d'expliquer en quelques mots ce qu'est le calque.

Nous commençons, d'abord, par la définition de calque figurant dans Le Petit Robert électronique qui le caractérise comme une «traduction littérale (d'une expression complexe ou d'un mot en emploi figuré) dans une autre langue ». C'est une définition simplifiée que nous pouvons élargir, parce qu'il peut ne pas s'agir seulement d'une difficulté de traduction des expressions complexes et des termes au sens figuré, mais de n'importe quelle structure d'une langue.

Dans le Dictionnaire de linguistique et sciences du langage, l'article « calque » explicite que ce type de formation est devenu productif en langue française :

Quand il s'agit d'un mot simple, le calque se manifeste par l'addition, au sens courant du terme, d'un « sens » emprunté à la langue $\mathrm{B}$; ainsi, le mot réaliser, dont le sens est « rendre réel, effectif », a pris aussi celui de " comprendre » (Il a réalisé la situation) par calque de l'anglais to realize. Quand il s'agit d'un mot composé, la langue A conserve souvent l'ordre des éléments de la langue $\mathrm{B}$, même lorsque cet ordre est contraire à celui que l'on observe ailleurs dans l'usage de la langue ; ainsi gratte-ciel est formé des mots français gratte et ciel, mais c'est un calque de l'anglo-américain sky-scraper, dont il a modifié l'ordre pour se conformer à la syntaxe du français; en revanche, quartier-maître est formé des mots quartier et maître, mais c'est un calque de l'allemand Quartiermeister, dont il conserve l'ordre (alors que, en français, le déterminant quartier devrait suivre le déterminé maître. (DUBOIS et al., 2007, p. 74)

À partir de la définition apportée par Le Petit Robert et des exemples fournis par le Dictionnaire de linguistique et sciences du langage, nous pouvons concevoir le calque comme un emprunt structurel. Autrement dit, le calque représente une tentative de reproduction fidèle d'une structure de la langue-source dans la langue-cible. La réalisation d'un calque peut être acceptable, comme les cas exposés ci-dessus le montrent, ou fautive, comme nous le démontrons par le moyen des exemples que nous examinons dans cet article.

Pour illustrer la question à laquelle nous nous intéressons, nous exposons un cas de calque fautif dans le domaine de la syntaxe : il s'agit de l'expression du pronom sujet de façon 

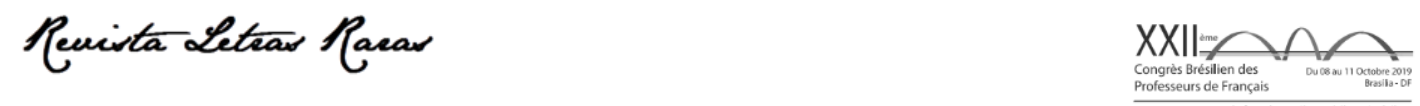

Actes du XXIlème Congrès brésilien des professeurs de français (dans Édition spéciale de la Revue Letras Raras 2020)

ISSN : 2317-2347 - v. 9, Dossier Spécial (2020)

Todo o conteúdo da RLR está licenciado sob Creative Commons Atribuição 4.0 Internacional

excessive dans la traduction de langues de paramètre non pro-drop ${ }^{1}$, c'est-à-dire des langues ne permettant pas l'omission de certains pronoms comme l'anglais et le français, en langues pro-drop, comme le portugais et la presque totalité de langues romanes. Dans ce sens, différemment de la langue française, la langue portugaise permet l'omission du pronom sujet dans une proposition lorsqu'il est grammaticalement ou pragmatiquement inférable. Observons ci-dessous (Tab 1) un exemple concret de l'expression excessive d'un élément anaphorique :

Tableau 1: Exemple de calque français $\rightarrow$ portugais

Elle s'appelle Marie. Elle est professeur d'anglais. Elle aime la littérature et elle adore le théâtre.

Ela se chama Maria. Ela é professora de inglês. Ela gosta de literatura e ela adora o teatro.

Source : élaboré par les auteurs de cet article.

Comme on peut le remarquer dans le tableau ci-dessus, l'extrait en français montre tous les verbes (" s'appeler », " être », " aimer » et « adorer ») précédés du pronom sujet " elle ». Dans la traduction mot-à-mot en portugais, le pronom ela précède également les verbes dans chacune des propositions, ce qui caractérise l'occurrence d'un calque.

L'emploi des structures calquées est susceptible de provoquer des erreurs, voire des contradictions dans la traduction. À ce propos, Paulo Rónai note que : « L'ordre des mots dans la phrase est un élément constitutif de son sens. Pour cette raison, donner tout simplement l'équivalent des mots figurant dans le texte original, quelle que soit la langue, ne sera jamais une traduction »(1983a, p. 4, c'est nous qui traduisons) $)^{2}$. C'est-à-dire qu'une traduction mot-à-mot peut susciter des divergences en ce qui concerne les constructions, la signification et les valeurs se trouvant dans la version originale d'un texte, des éléments qui doivent être bien compris et respectés.

\section{La traduction de Véra et le calque}

\footnotetext{
${ }^{1}$ Réduction de pronom-dropping, en anglais, et dont le sens est « suppression du pronom ».

2 « a ordem das palavras na frase é um expediente que entra a compor sentido. Por isso mesmo, enfileirar simplesmente os equivalentes das palavras do original em qualquer outra língua não será nunca tradução » (1983a, p. 4). La traduction en français des citations - dont les originaux figurent dans des notes de bas de page - a été réalisée par les auteurs de cet article.
} 

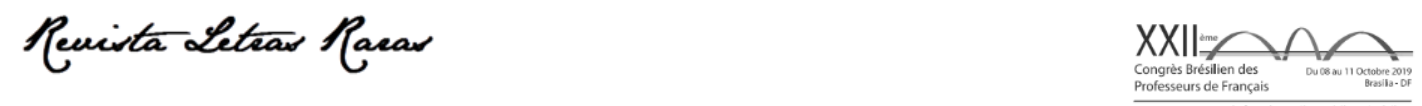

Actes du XXIlème Congrès brésilien des professeurs de français (dans Édition spéciale de la Revue Letras Raras 2020)

ISSN : 2317-2347 - v. 9, Dossier Spécial (2020)

Todo o conteúdo da RLR está licenciado sob Creative Commons Atribuição 4.0 Internacional

Pour procéder à une traduction juste et cohérente, nous avons observé les stratégies employées par Villiers de l'Isle-Adam relativement à la forme et au contenu afin de réfléchir aux équivalents les plus adéquats, tenant compte du ton et du registre rencontrés dans le texte en langue-source.

Véra est un court récit fantastique français qui raconte, par le moyen d'un narrateur hétérodiégétique (narrateur ne participant pas à l'action), l'histoire du comte Roger D'Athol qui n'accepte pas la mort prématurée de son épouse, Véra. Roger s'isole dans un manoir parisien dans la deuxième moitié du XIXe siècle et vit une existence insolite à côté du fantôme supposé de sa bien-aimée.

Avant de commencer le processus traductif, nous avons analysé la nouvelle de Villiers de I'Isle-Adam par le moyen d'une perspective diégétique, discursive et thématique. Cet examen nous a aidées à mieux comprendre le contexte et, par conséquent, à surmonter la première grande difficulté de compréhension : le vocabulaire technique courant au XIXe siècle et qui n'est plus en usage au XXle siècle (comme quelques expressions relevant du domaine de l'architecture, de la décoration, des vêtements, etc.). Remarquons, pourtant, que même la lecture la plus prudente permet parfois uniquement de déduire ou d'imaginer la valeur de tel ou tel mot dans le texte et, comme l'a signalé Paulo Rónai : « Comprendre approximativement un texte n'est pas le traduire. Les difficultés surviennent lorsque nous cherchons à le transposer par écrit, tout en essayant d'interpréter chacun de ses mots avec exactitude » (1983b, p. XI, c'est nous qui traduisons) ${ }^{3}$.

Après avoir réalisé la première version de la traduction de Véra, nous nous sommes rendu compte, au cours de sa révision, de l'influence de quelques structures de la langue française dans le texte traduit : nous étions, dans ce sens, en présence de quelques exemplaires de calque.

\subsection{Le calque et la morphosyntaxe : le cas du plus-que-parfait}

Le premier exemple de calque que nous fournissons a été constaté dans le domaine de la morphosyntaxe et relève de la transposition du plus-que-parfait de l'indicatif, en langue française, au mais-que-perfeito do indicativo, en langue portugaise. Dans quelle mesure cette transposition pourrait-elle représenter un calque?

\footnotetext{
3 « compreender aproximadamente um texto não é traduzi-lo. As dificuldades repontam quando começamos a transpô-lo por escrito, tentando interpretar com exatidão cada palavra » (1983b, p. XI).
} 

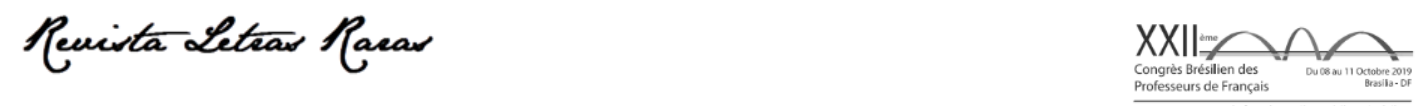

Actes du XXIlème Congrès brésilien des professeurs de français (dans Édition spéciale de la Revue Letras Raras 2020) ISSN : 2317-2347 - v. 9, Dossier Spécial (2020)

Todo o conteúdo da RLR está licenciado sob Creative Commons Atribuição 4.0 Internacional

Il convient tout d'abord de préciser la divergence existant entre la forme du plus-que-parfait et les deux formes du mais-que-perfeito. En langue française, le plus-que-parfait se constitue d'une forme composée : un auxiliaire, « être » ou « avoir » à l'imparfait, plus un participe passé (ex. : « elle avait voyagé »). En langue portugaise, en revanche, le mais-que-perfeito possède deux formes : l'une simple, employée couramment dans des situations discursives formelles et, surtout, dans le domaine écrit (ex. : Ele cantara, en français « il avait chanté »), et l'autre composée, employée dans des situations informelles, mais de plus en plus également dans des situations que l'on peut qualifier de formelles (ex. : Ele tinha/havia cantado, en français « il avait chanté »). De nos jours, la forme simple du plus-que-parfait est observée, surtout, dans des textes ayant un registre littéraire et/ou soutenu.

Dans Véra, il n'y a pas seulement l'idée d'un formalisme, exprimé dans le récit rapporté par le narrateur hétérodiégétique, mais aussi un langage dénotant un soin et indiquant un registre soutenu. Dans la première version de notre traduction, nous avons traduit mot-à-mot le plus-queparfait, c'est-à-dire qu'en raison de sa forme composée dans la langue-source, le français, nous avons utilisé cette même structure dans notre traduction en portugais du Brésil.

Observons la première version de l'exemple 1 (Tab. 2) :

Tableau 2: Exemple 1/Nersion 1

\begin{tabular}{|l|l|l|}
\hline \multicolumn{1}{|c|}{ Français } & & \multicolumn{1}{|c|}{ Portugais du Brésil } \\
\hline $\begin{array}{l}\text { "La Mort, subite, avait foudroyé. La nuit } \\
\text { dernière, sa bien-aimée s'était évanouie }\end{array}$ & & $\begin{array}{l}\text { A Morte, súbita, tinha fulminado. Na noite } \\
\text { anterior, sua bem-amada tinha perdido os } \\
\text { en des joies si profondes, s'était perdue en } \\
\text { de si exquises étreintes, que son cœur, } \\
\text { brisé de délices, avait défailli : ses lèvres } \\
\text { s'étaient brusquement mouillées d'une }\end{array}$ \\
\hline $\begin{array}{l}\text { pourpre mortelle. » (VILLIERS DE L'ISLE- } \\
\text { ADAM, 1986, p. 553-554) }\end{array}$ & V1 & $\begin{array}{l}\text { se perdido em carícias tão deliciosas, que } \\
\text { seu coração, estilhaçado de delícias, tinha } \\
\text { desfalecido: seus lábios tinham se } \\
\text { molhado subitamente de um carmesim } \\
\text { mortal. }\end{array}$ \\
\hline
\end{tabular}

Source : élaboré par les auteurs de cet article.

À l'occasion de la révision, nous avons constaté que ce choix ne serait pas le plus adéquat si nous tenions compte du registre employé dans le texte original; nous avions alors commis un calque du français. Nous avons remarqué que pour conserver le ton et le registre du texte-source, nous devrions utiliser une autre structure verbale. C'est la raison pour laquelle nous avons remplacé les occurrences du plus-que-parfait dans la deuxième version de la traduction sous la forme simple du mais-que-perfeito (Tab. 3). 

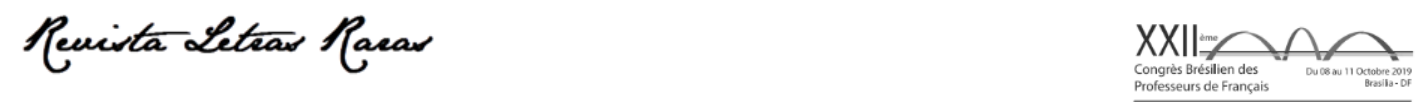

Actes du XXIlème Congrès brésilien des professeurs de français (dans Édition spéciale de la Revue Letras Raras 2020)

ISSN : 2317-2347 - v. 9, Dossier Spécial (2020)

Todo o conteúdo da RLR está licenciado sob Creative Commons Atribuição 4.0 Internacional

Tableau 3: Exemple 1/Nersion 2

\begin{tabular}{|c|c|c|}
\hline Français & & Portugais du Brésil \\
\hline $\begin{array}{l}\text { "La Mort, subite, avait foudroyé. La nuit } \\
\text { dernière, sa bien-aimée s'était évanouie } \\
\text { en des joies si profondes, s'était perdue en } \\
\text { de si exquises étreintes, que son cœur, } \\
\text { brisé de délices, avait défailli : ses lèvres } \\
\text { s'étaient brusquement mouillées d'une } \\
\text { pourpre mortelle » (VILLIERS DE L'ISLE- } \\
\text { ADAM, 1986, p. } 553 \text { - } 554 \text { ). }\end{array}$ & V2 & $\begin{array}{l}\text { A Morte, súbita, fulminara. Na noite } \\
\text { anterior, sua bem-amada perdera os } \\
\text { sentidos em alegrias tão profundas, } \\
\text { perdera-se em carícias tão deliciosas, que } \\
\text { seu coração, partido de delícias, } \\
\text { desfalecera: seus lábios molharam-se } \\
\text { subitamente de um carmesim mortal. }\end{array}$ \\
\hline
\end{tabular}

Source: élaboré par les auteurs de cet article.

Les constructions "avait foudroyé », "s'était évanouie », "s'était perdue », " avait défailli » et " s'étaient mouillées », traduites a priori par tinha fulminado, tinha perdido, tinha se perdido, tinha desfalecido et tinham se molhado, ont été traduites, dans la deuxième version, par fulminara, perdera, perdera-se, desfalecera et molharam-se.

Notons que la situation se répète dans l'exemple 2 (Tab. 4).

Tableau 4: Exemple 2/Nersion 1

\begin{tabular}{|l|l|l|}
\hline \multicolumn{1}{|c|}{ Français } & & \multicolumn{1}{|c|}{ Portugais du Brésil } \\
\hline $\begin{array}{l}\text { «Le serviteur pensa d'abord que la douleur } \\
\text { trop lourde, trop désespérée, avait égaré } \\
\text { l'esprit de son maître » (VILLIERS DE } \\
\text { L'ISLE-ADAM, 1986, p. 557). }\end{array}$ & V1 & $\begin{array}{l}\text { O criado pensou primeiramente que a dor } \\
\text { tão forte, tão desesperada, havia } \\
\text { desvairado o espírito de seu mestre. }\end{array}$ \\
\hline
\end{tabular}

Source : élaboré par les auteurs de cet article.

La construction verbale « avait égaré » a été traduite par havia desvairado, tout en gardant la structure de la langue-source. Cependant, comme cet extrait se trouve dans le domaine du récit et non pas dans celui des dialogues entre les personnages, le plus approprié était la forme simple de ce temps verbal, comme nous l'exposons ci-dessous (Tab. 5) :

Tableau 5: Exemple 2/Nersion 2

\begin{tabular}{|l|l|l|}
\hline \multicolumn{1}{|c|}{ Français } & & \multicolumn{1}{|c|}{ Portugais du Brésil } \\
\hline $\begin{array}{l}\text { «Le serviteur pensa d'abord que la douleur } \\
\text { trop lourde, trop désespérée, avait égaré } \\
\text { l'esprit de son maître » (VILLIERS DE } \\
\text { L'ISLE-ADAM, 1986, p. 557). }\end{array}$ & V2 & $\begin{array}{l}\text { O criado pensou, primeiramente, que a dor } \\
\text { tão pesada, tão desesperada, desvairara o } \\
\text { espírito de seu mestre. }\end{array}$ \\
\hline
\end{tabular}

Source : élaboré par les auteurs de cet article.

Pour cette raison, au moment de notre révision, nous avons choisi desvairara au détriment de havia desvairado. Après avoir remplacé les structures composées du mais-que-perfeito par leur 

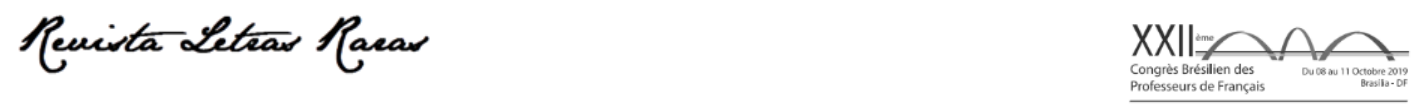

Actes du XXIlème Congrès brésilien des professeurs de français (dans Édition spéciale de la Revue Letras Raras 2020)

ISSN : 2317-2347 - v. 9, Dossier Spécial (2020)

Todo o conteúdo da RLR está licenciado sob Creative Commons Atribuição 4.0 Internacional

forme simple, nous avons réussi à mieux représenter ce temps verbal pour ce qui est du ton et notamment du registre.

\subsection{Le calque et la stylistique}

La deuxième question que nous présentons appartient au domaine de la morphosyntaxe, comme de la stylistique dans le cadre de la traduction littéraire. L'exemple 3 (Tab. 6) porte sur la devise/le jeu de mots "Qui verra Véra l'aimera », dont la sonorité est fluide grâce à un effet d'assonance. Nous savons qu'un jeu de mots peut être assez significatif en raison des effets esthétiques qu'il peut attribuer à un texte littéraire, il est donc important de les maintenir dans la langue-cible au moment de reáliser sa traduction.

Toutefois, dans la première version de notre traduction, la structure originale de l'expression a été calquée, c'est-à-dire que nous l'avons traduite mot-à-mot, ce qui a provoqué une équivoque se rapportant à la grammaire normative de la langue portugaise, tout comme une nuisance de la sonorité :

Tableau 6: Exemple 3/Version 1

\begin{tabular}{|l|l|l|}
\hline \multicolumn{1}{|c|}{ Français } & & \multicolumn{1}{c|}{ Portugais du Brésil } \\
\hline \begin{tabular}{l} 
" et, au pied du lit, sur une fourrure noire, \\
les petites mules de velours oriental, sur \\
lesquelles une devise rieuse de Véra \\
brillait, brodée en perles : Qui verra Véra \\
l'aimera » (VILLIERS DE L'ISLE-ADAM, \\
\hline 1986, p. 554).
\end{tabular} & V1 & $\begin{array}{l}\text { e, ao pé da cama, sobre um agasalho de } \\
\text { pele negro, os pequenos chinelos de veludo } \\
\text { oriental, sobre os quais uma divisa } \\
\text { sorridente de Vera brilhava, bordada em } \\
\text { pérolas: Quem verá Vera a amará. }\end{array}$ \\
\hline
\end{tabular}

Source : élaboré par les auteurs de cet article.

Le pronom a, en langue portugaise, placé avant le verbe entraîne une pause qui n'existe pas en français. De cette façon, au moment de la révision, cet aspect a été remarqué et nous avons reformulé la tournure comme le suit (Tab. 7):

Tableau 7: Exemple 3/Version 2

\begin{tabular}{|l|l|l} 
Français & & Portugais du Brésil \\
\hline
\end{tabular}



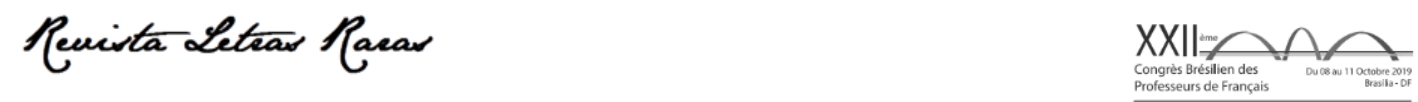

Actes du XXIlème Congrès brésilien des professeurs de français (dans Édition spéciale de la Revue Letras Raras 2020)

ISSN : 2317-2347 - v. 9, Dossier Spécial (2020)

Todo o conteúdo da RLR está licenciado sob Creative Commons Atribuição 4.0 Internacional

« et, au pied du lit, sur une fourrure noire, les petites mules de velours oriental, sur lesquelles une devise rieuse de Véra brillait, brodée en perles : Qui verra Véra l'aimera » (VILLIERS DE L'ISLE-ADAM, 1986, p. 554).

Source : élaboré par les auteurs de cet article.

En portugais du Brésil, quand le verbe est conjugué aux temps du futur de l'indicatif (futuro do presente et futuro do pretérito), il faut placer le pronom complément entre le radical du verbe et sa désinence ; cette règle s'appelle mesóclise et elle appartient à un registre soutenu. Ainsi, en vue d'une rectification, nous avons remplacé à amará par amá-la-á, ce qui a également changé la sonorité de l'expression, rendant celle-ci plus proche de la construction employée dans le texte original. Nous avons, dans ce sens, corrigé une erreur en langue portugaise et contribué à une meilleure transposition du jeu de mots trouvé dans l'expression « Qui verra Véra l'aimera ».

\section{En guise de conclusion}

Le travail de traduction d'un ouvrage littéraire suscite d'importantes réflexions théoricopratiques et l'identification de quelques situations traductives qui doivent être examinées, comme celles exposées dans cet article. Face à ces obstacles, nous avons besoin d'associer la théorie et la pratique pour chercher des réponses dans différentes sources (des dictionnaires généraux de la langue française et portugaise, des dictionnaires de linguistique, des dictionnaires spécialisés, etc.).

Grâce aux études de Paulo Rónai, nous avons pu réfléchir à une série de difficultés dans le processus de traduction des textes littéraires. Ainsi, nous avons pris connaissance des pièges les plus courants, ce qui nous a permis d'être plus attentives.

Dans cet article, nous avons exposé deux questions liées au calque apparaissant dans la traduction du français en portugais : soit dans le domaine de la morphosyntaxe, exemplifiée par la forme du plus-que-parfait/mais-que-perfeito, soit dans le domaine de la stylistique, exemplifiée par un jeu de mots. Ce sont deux problématiques importantes quand nous pensons au lecteur brésilien et à la lecture fluide et claire du texte dans sa forme traduite.

Pour conclure, nous avons réfléchi à l'impossibilité d'une traduction littérale (mot-à-mot) dans la plupart des cas, étant donné que les mots ne sont pas pensés et rédigés d'une façon 

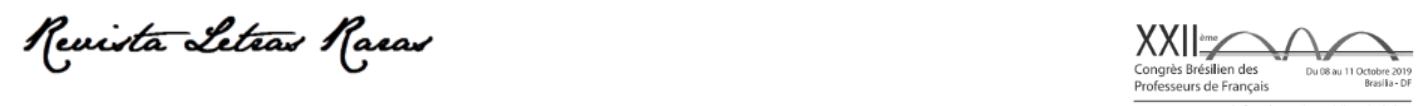

Actes du XXIlème Congrès brésilien des professeurs de français (dans Édition spéciale de la Revue Letras Raras 2020)

ISSN : 2317-2347 - v. 9, Dossier Spécial (2020)

Todo o conteúdo da RLR está licenciado sob Creative Commons Atribuição 4.0 Internacional

indépendante, mais dans un contexte. Une traduction hors contexte est un danger pour la compréhension d'une œuvre littéraire comme de n'importe quel genre de texte, ainsi il faut souligner que "Le bon traducteur, après s'être renseigné sur le contenu d'un énoncé, essaie d'oublier les mots utilisés, pour rechercher ensuite dans sa langue les mots qui permettraient d'exprimer la même idée » (RÓNAI, 1976, p. 33, c'est nous qui traduisons)4. Autrement dit, le traducteur doit transposer en langue-cible, avant tout, le sens et l'intention de l'auteur traduit, même si, pour autant, il lui faudra se servir de structures partiellement ou totalement différentes de celles rencontrées dans le texte original.

\section{Références}

DUBOIS, J. et al. Grand dictionnaire - Linguistique \& sciences du langage. Paris : Larousse, 2007. ROBERT, P. Le Petit Robert électronique. CD-ROM. Paris : Dictionnaires Le Robert, 2014.

RÓNAl, P. A tradução vivida. Rio de Janeiro: Educom, 1976.

RÓNAI, P. Problemas gerais da tradução. In: RÓNAI, P. A tradução técnica e seus problemas. São Paulo: Álamo, 1983a, p. 1-16.

RÓNAI, P. Guia Prático da Tradução Francesa: relação alfabética dos falsos amigos, homônimos, parônimos, cognatos de gêneros diferentes. $4^{\mathrm{a}}$ ed. rev. e ampl. Rio de Janeiro: Nova Fronteira, $1983 b$.

VILLIERS DE L'ISLE-ADAM, A. ; RAITT, A. ; CASTEX, P-G. et al. CEuvres complètes. Paris: Gallimard, 1986. Bibliothèque de la Pléiade.

\footnotetext{
${ }^{4}$ « o bom tradutor, depois de se inteirar do conteúdo de um enunciado, tenta esquecer as palavras em que ele está expresso, para depois procurar, na sua língua, as palavras exatas em que semelhante ideia seria naturalmente vazada »(RÓNAl, 1976, p. 33).
} 